\title{
Associations of Probiotics with Vitamin D and Leptin Receptors and their Effects on Colon Cancer
}

\author{
Peyman Ranji ${ }^{1}$, Abolfazl Akbarzadeh ${ }^{2,3}$, Mohammad Rahmati-Yamchi ${ }^{2,3 *}$
}

\begin{abstract}
Colorectal cancer (CRC) is one of most common causes of cancer-related death worldwide. Recent studies have suggested that microbial and environmental factors including diet and lifestyle can impact on colon cancer development. Vitamin D deficiency and dysfunction of vitamin D receptor (VDR) also correlate with colon cancer. Moreover, leptin, a 16-kDa polypeptide, participates in the regulation of food intake and is associated with other environmental factors affecting colon cancer through the leptin receptor. Altered levels of serum leptin and patterns of expression of its receptor (LPR) may be observed in human colon tumours. Furthermore, the collected data from in vitro and in vivo studies have indicated that consuming probiotic non-pathogenic lactic acid bacteria have beneficial effects on colon cancer. Probiotics, inflammation and vitamin D/VDR have been correlated with leptin and its receptor and are also with colon cancer. Thus, in this paper, we review recent progress on the roles of probiotic, vitamin D/VDR and leptin/LPR in inflammation and colon cancer.
\end{abstract}

Keywords: Colon cancer - leptin - leptin receptor - probiotic - vitamin D - vitamin D receptor

Asian Pac J Cancer Prev, 16 (9), 3621-3627

\section{Introduction}

Colorectal cancer (CRC) is one of most common causes of cancer-related death worldwide (Yu et al., 2014). Recent studies have suggested that microbial and some environmental factors including diet and lifestyle, can induce colon cancer (Slattery et al., 2007). Moreover, Imbalance between different species of gut bacteria is assumed to be related with CRC risk factors such as inflammatory bowel diseases (IBD) .Among other environmental factors such as diet, Vitamin D is important factor and regulates reaction to intestinal immune responses, anti-inflammation, and cancer inhibition via vitamin D receptor (VDR). Also, bacterial density and growth effect on VDR expression (Lu et al., 2012). Leptin polypeptide and leptin receptor (LPR) are other important factor that participate in the regulation of food intake . Leptin and LPR might be implicated in the modulation of inflammation and CRC progression (Joshi and Lee, 2014). There is growing evidence that nuclear hormone receptors such as the vitamin D receptor, regulate the action of leptin and also Leptin has been shown to reduce expression of renal 25-hydroxyvitamin D (3)-1 $\alpha$-hydroxylase in mice via the leptin receptor (Menendez et al., 2001; Leung, 2004; Matsunuma and Horiuchi, 2007).

Probiotics are beneficial non-pathogenic lactic acid bacteria and have been consumed as food component or supplement in the inhibition or treatment of some diseases (Guarner and Malagelada, 2003). Inflammation and overgrowth of pathogenic organisms within the gastrointestinal tract, production of essential nutrients and/ or bioactive food components, and promotion of intestinal immunity seriously might be associated with the risk of developing neoplastic disease such as colon cancer (Davis and Milner, 2009). Therefore, microbes may influence multiple processes correlated with a change in cancer risk and consequently removal of the inflammatory bacteria by probiotic bacteria is one potential mechanism that may modulate disease severity (O'mahony et al., 2001). Consuming some probiotic such as Lactobacillus casei, demonstrated inhibition effect on colorectal tumor growth in patients (Ishikawa et al., 2005).

Energy balance, adiposity, inflammation and vitamin D have been correlated with leptin and its receptor and also are correlated with colon cancer (Slattery et al., 2003; Slattery et al., 2008). In this current review, we will focus on recent progress on the roles of vitamin $\mathrm{D}$, leptin and their receptors (VDR and LPR) in inflammatory and colon cancer. Moreover, we will review the probiotic effect on colon cancer and also two important related factors; VDR and LPR (Figure 1).

\section{Probiotics and Colon Cancer}

CRC is a complex disease and a most common worldwide cancer, and associated with advancing age

${ }^{1}$ Department of Medical Biotechnology, Faculty of Advanced Medical Sciences, International Branch of Tabriz University of Medical sciences (Aras), ${ }^{2}$ Drug Applied Research Center, ${ }^{3}$ Department of Medical Biotechnology, Faculty of Advanced Medical Sciences, Tabriz University of Medical sciences, Tabriz, Iran ${ }^{\star} E q u a l$ contributors *For correspondence: rahmati_bio@yahoo.com 


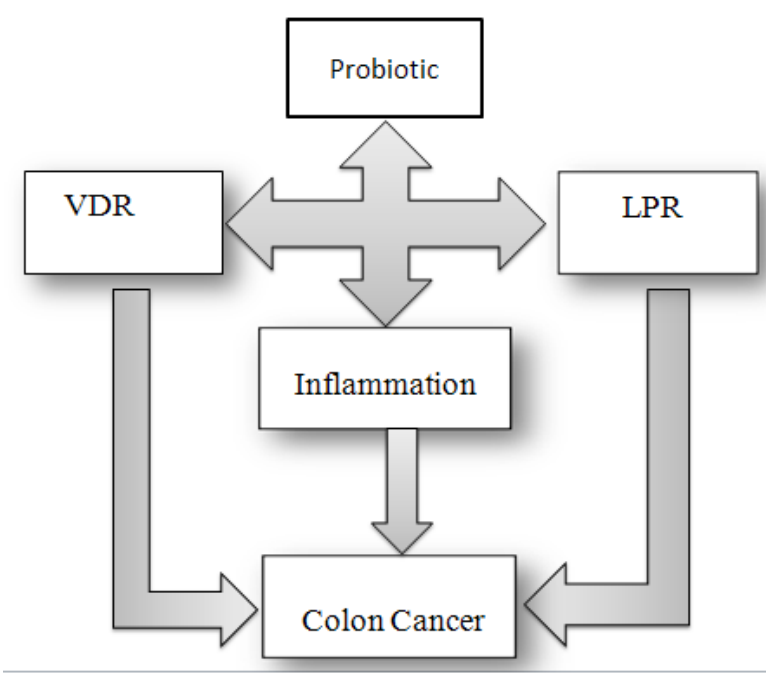

Figure 1. Vitamin D Receptor (VDR), Leptin Receptor (LPR) and Probiotic may be Critical Factors that Directly Contribute to Colon Cancer. Moreover, these factors can play a role on colon cancer through impacting on gut flora and inflammation. Furthermore, the collected data from studies demonstrated that there is association among VDR, LPR and probiotic. Thus, understanding molecular and genetic association among them and colon cancer can have beneficial effect on prevention and treatment of colon cancer

(Kumar et al., 2013). Genetic and environmental factors are two main important factors for CRC occurrence (Zhu et al., 2011). Mutations in tumor-suppressor genes such as Adenomatous polyposis coli (APC), catenin (cadherinassociated protein) beta 1, tumor protein p53 (CTNNB1, p53) and oncogenes Kirsten rat sarcoma viral oncogene homolog (KRAS), lead to initiating CRC (Fearon, 2011; Abreu and Peek, 2014). Moreover, Recent studies have suggested that microbial and environmental factors, such as diet and lifestyle, can affect and induce colon cancer (Slattery et al., 2007). Intestinal bacteria flora impact the immune system, induce cell growth, supply key nutrients, inhibit the growth of harmful microorganisms, modulate energy metabolism, and protect against diseases. Some bacteria such as Clostridium septicum, Streptococcus infantariusetc have pathogens roles associated with colon carcinoma in many case reports (Mirza et al., 2009; Lu et al., 2012). Imbalance between different species of enteric bacteria is assumed to be related with IBD and other CRC risk factors (Lu et al., 2012). It is not clear which specific microbes are particularly pathogenic and directly lead to colorectal carcinogenesis. Identification of single type of microbe among myriad of microbes that present in the human colon and cause cancer, would be a challenge because the intestinal microbiota change during disease development and progression (Al-Jashamy et al., 2010; Abreu and Peek, 2014).

The groups of microflora bacteria, as beneficial bacteria, have useful function in human health. Probiotics are beneficial non-pathogenic lactic acid bacteria and have been consumed as food component or supplement in the inhibition or treatment of some diseases (Davis and Milner, 2009; Modarressi et al., 2014). Two famous genera of beneficial bacteria involve bifidobacterium and lactobacillus that have therapeutic and health benefits including: (i) immune enhancement against intestinal infections; (ii) prevention of diarrhoeal diseases, hypercholesterolaemia and upper gastrointestinal tract diseases; (iii) improvement in lactose utilization; (iv) stabilization of the gut mucosal barrier and (v)prevention of colon cancer (Kailasapathy and Chin, 2000; Modarressi et al., 2014; Srinarong et al., 2014). Preclinical studies have demonstrated that consuming probiotics suppresses and inhibits CRC via influencing innate immune pathways and apoptosis, changing colonic motility and transit time, modulating intestinal bacteria and their metabolism , inhibition of carcinogens and/or procarcinogens and reducing oxidative stress (Zhu et al., 2011; Lee et al., 2013; Modarressi et al., 2014). A previous in vitro study demonstrated that Bacillus polyfermenticus SCD inhibited the growth of Caco-2 cells in a dose dependent manner (Lee et al., 2007). Moreover, anticancer activity of Bifidobacterium adolescentis SPM0212 was observed on proliferation of three human colon cancer cell lines: HT-29, SW 480, and Caco-2. Furthermore, in vivo studies showed that daily oral administration of the microencapsulated Lactobacillus acidophilus caused significant suppression of colon tumor incidence, tumor multiplicity, and reduced tumor size in 1, 2-dimethylhydrazine (DMH)-induced colon cancer in BALB/c mice and cellular apoptosis increased during the treatment (Urbanska et al., 2009; Zhu et al., 2011). Despite these laboratory-based studies, only a limited number of clinical trials have operated to evaluate the probiotics roles in suppressing CRC (Abreu and Peek, 2014). Lactobacillus species are the most usually applied probiotics in clinical trials. For example, Lactobacillus johnsonii decreased the concentration of Enterobacters and modulated intestinal immune response in CRC patients, whereas Bifidobacterium longum did not have any influence (Gianotti et al., 2010; Abreu and Peek, 2014). In another study, after 2-4 years of Lactobacillus casei consuming, suppression of colorectal tumor growth in patients was observed (Ishikawa et al., 2005). in addition, different probiotic bacteria have different effects and for this reason, specific signaling pathways of probiotic bacterial strains must be evaluated separately (O’mahony et al., 2001).

\section{Nuclear Receptor Superfamily and VDR}

The nuclear receptors (NRs) are a family of transcription factors that bind and respond to certain signaling molecules such as vitamin D3 and thyroid hormone. In recent years, newly recognized and more diverse ligands such as phospholipids and heme have been identified for the nuclear receptors (Sonoda et al., 2008; McEwan, 2009; Huang et al., 2010). NR superfamily can be divided into 3 groups according to ligand affinities. The first group of receptors, e.g. sex steroid and thyroid hormone receptors, vitamin D receptor (VDR), retinoic acid and retinoid X receptors binds to specific ligands with high affinity. The second group of receptors such as PPAR, liver X receptors and farnesoid $\mathrm{X}$ receptor bind to specific compounds such as PUFA and bile acids. Finally, orphan receptors group that no functional ligand-binding domain or no ligands have been identified as yet (Kotnis et al., 2005; Thorne and Campbell, 2008). The common structure of nuclear 
receptors includes DNA-binding domain (DBD), ligandbinding domain (LBD) and cofactor interactions. The variable region, termed as hinge region, link DBD to LBD. The DBD recognizes specific response elements $(\mathrm{RE})$ in the enhancer-promoter regions of target genes (Collins et al., 2005; Reya and Clevers, 2005; Thorne and Campbell, 2008; Huang et al., 2010). Most receptors specially make homo- or heterodimer complexes with other receptors .For example; retinoid $\mathrm{X}$ receptor forms heterodimer complexes with VDR, PPAR, liver $\mathrm{X}$ receptors and farnesoid X receptor (Thorne and Campbell, 2008; Patel et al., 2012). The binding of physiological ligands to nuclear receptors act simply as molecular messengers signaling and effect on body's physiological states. In the first step, these ligands diffuse in cells through specific signaling pathway and bind to LBD of NRs. In the second step, the ligand binding inside the LBD, leads to allosteric changes on the receptor surface. Finally, DNA response elements cause the physiological responses to ligands into an accurate genetic program (Hardwick et al., 2001; Huang et al., 2010). Identification of ligands with gene-selective actions and also impacting of these ligands on disease outcomes through a narrow subset of target genes has been the major challenge due to multitude of genes under the control of a single receptor (Mencarelli et al., 2011).

Vitamin D3 with important biological effects is synthesized in human skin with sunlight energy; and biological effects of vitamin D3, especially active form 1,25-dihydroxy vitamin $\mathrm{D}(1,25(\mathrm{OH}) 2 \mathrm{D} 3)$, the mostly are mediated by VDR. Vitamin D3 has important functions such as modulation of calcium homeostasis and regulation of blood pressure, metabolic syndrome, and inflammation (Lu et al., 2012; Yu et al., 2014). In human, VDR gene locates on the chromosome 12 and has high expression in metabolic tissues including skin, kidney, intestine, and thyroid gland, and moderately expression almost in all other tissues. Vitamin D3 binds to the VDR and then forms VDR and retinoid $\mathrm{X}$ receptor heterodimer complex that binds cooperatively to vitamin $\mathrm{D}$ responsive elements and regulates the transcription of target genes (Ramagopalan et al., 2010; Patel et al., 2012). Many vitamin D target genes such as p21,p27, and e-cadherin have been found to control cell cycle arrest and cell differentiation. Therefore, it has been proposed that $1 \alpha 25(\mathrm{OH} 2) \mathrm{D} 3$ through the VDR and up-regulating the above genes, can possibly be used for therapeutic cancer (Patel et al., 2012; Stubbins et al., 2012).

\section{Vitamin D, VDR and Inflammation-related Colon Cancer}

Imbalance between different species of enteric bacteria is assumed to be related with IBD or CRC risk factors. $\mathrm{CRC}$ increase in patients with long-term IBD and is accounts for $20 \%$ of IBD-related mortality (Basseri et al., 2011; Lu et al., 2012). Vitamin D/VDR controls reaction to intestinal immune responses, anti-inflammation, and cancer inhibition but the function of vitamin D/VDR in regulating bacterial flora in tumorigenesis remains unidentified. Moreover, microorganism infection can disable or downregulate the activity of VDR in related signaling pathway. For example, Borrelia burgdorferi was reported with reduction VDR expression in monocytes and Shigella infection limits the gene transcription ability of VDR by upregulation caspases (Suzuki et al., 2007; Kaji et al., 2010; Lu et al., 2012). Other study showed that commensal and pathogenic bacteria directly control expression of VDR in colonic epithelial. Furthermore, bacterial density and growth effect on VDR expression and its expression is higher in the proximal colon than in the distal colon (Wu et al., 2010; Lu et al., 2012). Furthermore, vitamin D/VDR has a function for intestinal homeostasis and host protection from infection and also has a target genes of VDR such as enzyme Cyp24 and antimicrobial peptides (AMP) such as cathelicidin and $\beta$ defensin. When the immune system is faced with pathogens, transforming growth factor $\beta$ (TGF- $\beta$ ) and interferon gamma (IFN- $\gamma$ ) are released and consequently, VDR is activated to express more cathelicidin and defensin that regulate the composition of bacterial flora. Furthermore, VDR is related with toll-like receptors (TLRs), which invoke the immune system for recognition bacteria (Carlberg and Dunlop, 2006; Schauber et al., 2007; Lu et al., 2012). Decreased vitamin D/VDR levels and change number and diversity of gut bacteria will promote inflammation and allergen sensitization (Weiss, 2011). Vitamin D directly modulates the T-cell receptor (TCR), and also downregulates the expression of proinflammatory cytokines. Moreover, vitamin D regulate function of autophagy and different immune cells containing T cells, B cells, macrophages, dendritic cells, and epithelial cells (Sun, 2010; Wu and Sun, 2011). Vitamin D pathway is participant in innate immune system that is the body's first line of defense against bacterial pathogens (Lu et al., 2012). Moreover; VDR plays a crucial role in adaptive immune pathways and also primary target genes of vitamin D encode cytokines IL-2, IL-10, and IL-12B (Matilainen et al., 2010). Deficiency of 1,25(OH) 2D3 has been observed in patients with IBD and also the presence of $1,25(\mathrm{OH})$ 2D3 in animal models inhibited the progress of IBD (Sentongo et al., 2002; Abreu et al., 2004; Yoon and Sun, 2011). Polymorphisms in the VDR gene and expression are associated with status of IBD and development of colitis (Wada et al., 2009; Rasool et al., 2013; Yu et al., 2014). VDR dysfunction causes a reduction in innate immune activity and increases sensivity to additional infections that contribute to inflammation progression. So, survey the associations between VDR, bacterial infection, and colitis-associated colon cancer can be helpful and main challenge (Lu et al., 2012).

\section{Vitamin D, VDR and Colon Cancer}

Recent epidemiological and experimental studies demonstrate that vitamin D deficiency associate with a large variety of human diseases, and especially with the high risk of colorectal cancer. Dysfunction of VDR and vitamin D3 deficiency can lead to poor bone development and health, increase the risk of many chronic diseases, such as rheumatoid arthritis, type 1 diabetes, inflammatory bowel diseases (IBD), infectious diseases, and cancer (Holick, 2010; Wu and Sun, 2011; Lu et al., 2012; 
Akinci et al., 2014). Serum vitamin D3 levels, and VDR expression at mRNA and protein levels is significantly decreased in IBD patients (Abreu et al., 2004; Wada et al., 2009). Ulcerative colitis is a form of IBD and the study demonstrated that VDR expression in ulcerative colitis patients with CRC is lower than non-colon cancer patients (Wada et al., 2009). To sum up, the above data suggested that the VDR can play important role on CRC through its association and effect with IBD.

1,25(OH) 2D3 controls the detoxification metabolism, sensitises cells to apoptosis and inhibits the proliferation of cultured human colon carcinoma cells by regulating the expression of many genes via several mechanisms. Activation of VDR by vitamin D3 can prevent tumor cell proliferation by promoting differentiation in different cancer cell lines .Tumor cells don't be able to synthesize the active form of vitamin D and thus fail to respond for VDR-mediated vitamin D effects (Samuel and Sitrin, 2008; Akinci et al., 2014). Moreover, animal study results showed that $1,25(\mathrm{OH}) 2 \mathrm{D} 3$ and several of its analogues decrease intestinal tumorigenesis in animal models and sensitivity to carcinogen treatment increase in VDR knock-out mice (Zinser et al., 2005; Pereira et al., 2012). Another animal study demonstrated that VDR may have a suppressive role in intestinal tumor growth through inhibition of $\beta$-catenin activity (Zheng et al., 2012). Moreover, vitamin D inhibit the paracrine/ autocrine growth at the early stage of tumor progression and thus, high VDR expression has been reported in early stage of colorectal tumor progression (Bouillon et al., 2008). However, VDR expression is lost during tumor dedifferentiation because of VDR transcriptional repressor upregulation, SNAIL1, in this stage (Pendas-Franco et al., 2008). Many vitamin D target genes such as p21,p27, and e-cadherin have been found to control cell cycle arrest and cell differentiation (Palmer et al., 2001; Stubbins et al., 2012). Therefore, it has been proposed that $1 \alpha 25(\mathrm{OH} 2)$ D3 through the VDR and up-regulating the above genes, can possibly be used for therapeutic cancer (Palmer et al., 2001; Stubbins et al., 2012).

\section{LPR and Colon Cancer}

Leptin, 16-kDa polypeptide, participate in the regulation of food intake and exerts its histological action through the leptin receptor. Leptin receptors ,the same as IL-6 ,are members of the pro-inflammatory cytokine receptor class I superfamily and are involved in regulation serum leptin concentration acting as a carrier protein .Moreover, Leptin receptors expression are observed in normal colon epithelial cells of human subjects (Drew, 2012; Joshi and Lee, 2014). Leptin has been associated with obesity, regulation of energy homeostasis, reproductive factors, bone remodeling, insulin signaling, inflammatory response, and neuroendocrine function (Slattery et al., 2008). Studies have demonstrated that leptin also can induce expression of the inflammatory marker CRP (C-reactive protein) (Shamsuzzaman et al., 2004). There is growing evidence that nuclear hormone receptors such as the vitamin $\mathrm{D}$ receptor, regulate the action of leptin and other class I cytokines, and also vitamin D3 inhibit leptin secretion in human adipose tissue (Menendez et al., 2001; Leung, 2004). Moreover, Leptin has been shown to reduce expression of renal 25-hydroxyvitamin D (3)-1 $\alpha$-hydroxylase in mice via the leptin receptor (Matsunuma and Horiuchi, 2007). Leptin binds to the leptin receptor, and activate a tyrosine kinase such as Janus kinase (JAK2) to initiate downstream signaling of suppressors of cytokine signaling 1 and 3 (SOCS) and signal transducer and activator of transcription-3 (STAT). SOCS limits insulin signaling and also SOCS3 limit leptin signaling, and this association between leptin and SOCS is importance for cancer (Ahima and Osei, 2004; Slattery et al., 2008). Moreover, activation of major mitogenic signal transduction pathway elements, extracellular-signal-regulated kinase and Src/ phosphoinositide 3-kinase/protein kinase B are some other signal transduction pathway that associated with colon cancer and LPR (Drew, 2012).

Leptin can act as a growth factor promoting angiogenesis, and anti-apoptotic tumorigenic on different cancer cell lines (Endo et al., 2011; Mencarelli et al., 2011). Leptin expression may regulate the proliferation and invasiveness of tumors in gastric cancer, pituitary adenomas and breast cancer (Tutino et al., 2011). Enhanced expression of leptin and its tissue receptor were also correlated with hematogeneous metastasis or recurrence in distant organs and also increase invasiveness of pituitary adenomas tumors (Isono et al., 2003; Ishikawa et al., 2004). Adipose tissue dysfunction probably have a function role in the growth of CRC (Endo et al., 2011). According to critical role of leptin in cell proliferation, the hypothesis was suggested that leptin and its receptor might be implicated in the modulation of CRC progression and invasion (Tutino et al., 2011). Collected data from other studies demonstrated that polymorphisms in the leptin and LPR gene associate with obesity and cancer (Mu et al., 2014). The study demonstrated that the LPR is expressed in human colon cancer cells lines.The another study showed that expression of leptin and its receptor associate with grade of tumor differentiation, depth of bowel wall invasion, and distant metastasis in CRC patients. This study also recommended that the binding of leptin to its receptor enhance the proliferation of CRC (Joshi and Lee, 2014). Moreover, high circulating levels of LPR in patients is correlated with advanced stage of CRC (Hui et al., 2011; Tutino et al., 2011). The in vitro studies demonstrated that leptin induced cell proliferation and accompanying DNA synthesis in HT-29 and CACO-2 cell lines (Aparicio et al., 2005). Leptin is also observed to prevent apoptosis in human colon cancer cells through processes associated with extracellular-signal-regulated kinase, p38 mitogen- activated protein kinase activation and nuclear translocation of NF-kB (Hoda et al., 2007; Drew, 2012). Animal studies provide evidence that leptin stimulates proliferation of colon cells and could potentially increase sensitivity to two colon carcinogens, azoxymethane and $\mathrm{N}$-methylnitrosourea (Bartucci et al., 2010). Recent animal study reports that leptin plays a role in obesity-related cancer and increases colon tumour growth in obesity subsequent to the initiation of colon cancer. Leptin receptor expression increased in 
azoxymethane-treated mice and subsequently observed differences in tumour growth in colon tumours (Bartucci et al., 2010). Furthermore, the results of clinical studies demonstrated that altered patterns of leptin receptors expression were revealed in human colon tumours and LPR overexpression has been associated with better prognosis ,increased age, proximally located tumours, high levels of microsatellite instability and lymphocyte infiltration (Aloulou et al., 2008; Drew, 2012). Some clinical studies report decrease serum leptin in colon cancer patients, while others show that increased serum leptin is related to incidence of colon cancer in men, but not in women (Bolukbas et al., 2004; Kumor et al., 2009; Drew, 2012). Salageanu and his colleagues (2010) also report that serum leptin decrease in colon cancer patients and that levels decreased with tumour development and aggressiveness (Sălăgeanu, 2010). Furthermore, A more recent report failed to reveal significant alterations in serum leptin in colon cancer patients and controls (Uchiyama et al., 2011).

\section{Probiotic Effects on VDR and LPR}

According to above research results and explanations, in this part, we focused on probiotics effects on VDR and LPR, but there is a paucity of data on this field .Probiotic induced the modulation of anti-inflammatory VDR signaling in colitis but its mechanism remains virtually unknown. Recent studies show that in VDR-/- mice have been induced bacterial loading in the intestine and also stimulation of both commensal and pathogenic bacterial (Lagishetty et al., 2010; Wu et al., 2010). In the IL10-/colitis model was demonstrated that probiotic VSL3\# was associated with NR signaling pathway (Reiff et al., 2009; Yoon and Sun, 2011). The data collected from other study demonstrated probiotic treatment is able to increase VDR expression and activity in the host. Moreover, in vitro study show that treatment with Lactobacillus plantarum was leaded to a concomitant increase in VDR expression and cathelicidin mRNA (Yoon et al., 2011). In addition, intestinal VDR increased significantly after probiotic colonization in probiotic monoassociated pig model compared to the ex-germ-free pig (Yoon and Sun, 2011). in spite of that, probiotics did not inhibit inflammation in mice lacking VDR (Yoon and Sun, 2011). According to mentioned above, restoring vitamin D/VDR signaling may increase the host's ability to modulate inflammation in IBD and CRC patients (Lu et al., 2012). Moreover, leptin and LPR has been associated with inflammatory response and concentration of microflora bacteria (Slattery et al., 2008). Although the study demonstrated that VSL\#3 attenuates leptin production from mesenteric adipose tissue and creeping fat ,but considerable preclinical and clinical studies did not operate with probiotic effect on LPR (Mencarelli et al., 2011). Therefore, it seems that probiotic effect on LPR should be evaluated in preclinical and clinical studies in future. To sum up, understanding VDR's and LPR's contribution to probiotic induced anti-inflammation may provide significant insight in development of novel treatments in colitis-associated colon cancer and CRC.

\section{Conclusion and Future Directions}

Colorectal cancer (CRC) is one of most common causes of cancer-related death worldwide (Mencarelli et al., 2011). Recent studies have suggested that microbial and some environmental factors including diet and lifestyle, can induce colon cancer (Slattery et al., 2007). Imbalance between different species of gut bacteria is assumed to be related with CRC risk factors such as inflammatory bowel diseases (IBD) (Lu et al., 2012). Probiotics are beneficial non-pathogenic lactic acid bacteria and modulate intestinal bacteria and their metabolism. The collected data from in vitro and in vivo studies demonstrated that consuming probiotic has a beneficial effect on colon cancer. Moreover, VDR can play important role on CRC through its association and effect with IBD and colon cancer signaling pathway. There are limited genetic and clinical data in humans about vitamin D roles on colon cancer but this limited data suggest that vitamin $\mathrm{D}$ is protective against colon cancer. Beside this, leptin and LPR can act as a growth factor promoting angiogenesis, and antiapoptotic tumorigenic on different cancer cell lines such as colon cancer (Endo et al., 2011; Mencarelli et al., 2011). Moreover, energy balance, adiposity, inflammation and vitamin D have been correlated with leptin and its receptor and are also correlated with colon cancer (Slattery et al., 2003; Slattery et al., 2008). The above data suggested that understanding VDR's and LPR's contribution to probiotic induced anti-inflammation may provide significant insight in development of novel treatments in colitis-associated colon cancer and especially CRC. Thus, evaluation of the probiotic effect on colon cancer and also two important related factors; VDR and LPR, can be helpful and have beneficial effect on prevention and treatment of colon cancer. To sum up, these collected data show that limited preclinical and clinical studies operated in this field and molecular, genetic and clinical data in humans are scarce, thus future research on novel mechanisms of probiotics action on VDR and LPR is needed.

\section{References}

Abreu M, Kantorovich V, Vasiliauskas E, et al (2004). Measurement of vitamin D levels in inflammatory bowel disease patients reveals a subset of Crohn's disease patients with elevated 1,25-dihydroxyvitamin D and low bone mineral density. Gut, 53, 1129-36.

Abreu MT, Peek RM, Jr. (2014). Gastrointestinal malignancy and the microbiome. Gastroenterology, 146, 1534-46.

Ahima RS, Osei SY (2004). Leptin signaling. Physiology \& Behavior, 81, 223-41.

Akinci MB, Sendur M,Aksoy S, et al (2014). Serum 25-hydroxy Vitamin D status is not related to osteopenia/osteoporosis risk in colorectal cancer survivors. Asian Pac J Cancer Prev: APJCP, 15, 3377.

Al-Jashamy K, Murad A, Zeehaida M, et al (2010). Prevalence of colorectal cancer associated with Streptococcus bovis among inflammatory bowel and chronic gastrointestinal tract disease patients. Asian Pac J Cancer Prev, 11, 1765-8.

Aloulou N, Bastuji-Garin S, Le Gouvello S, et al (2008). Involvement of the leptin receptor in the immune response in intestinal cancer. Cancer Res, 68, 9413-22. 
Aparicio T, Kotelevets L, Tsocas A, et al (2005). Leptin stimulates the proliferation of human colon cancer cells in vitro but does not promote the growth of colon cancer xenografts in nude mice or intestinal tumorigenesis in ApcMin/+ mice. Gut, 54, 1136-45.

Bartucci M, Svensson S, Ricci-Vitiani L, et al (2010). Obesity hormone leptin induces growth and interferes with the cytotoxic effects of 5-fluorouracil in colorectal tumor stem cells. Endocrine-related Cancer, 17, 823-33.

Basseri RJ, Basseri B, Papadakis KA (2011). Dysplasia and cancer in inflammatory bowel disease.

Bolukbas FF, Kilic H, Bolukbas C, et al (2004). Serum leptin concentration and advanced gastrointestinal cancers: a case controlled study. BMC Cancer, 4, 29.

Bouillon R, Carmeliet G, Verlinden L, et al (2008). Vitamin D and human health: lessons from vitamin D receptor null mice. Endocrine Reviews, 29, 726-76.

Carlberg C, Dunlop TW (2006). The impact of chromatin organization of vitamin D target genes. Anticancer Research, 26, 2637-45.

Collins AT, Berry PA, Hyde C, et al (2005). Prospective identification of tumorigenic prostate cancer stem cells. Cancer research, 65, 10946-51.

Davis CD, Milner JA (2009). Gastrointestinal microflora, food components and colon cancer prevention. J Nutritional Biochemistry, 20, 743-52.

Drew JE (2012). Molecular mechanisms linking adipokines to obesity-related colon cancer: focus on leptin. Proceedings of the Nutrition Society, 71, 175-80.

Endo H, Hosono K, Uchiyama T, et al (2011). Leptin acts as a growth factor for colorectal tumours at stages subsequent to tumour initiation in murine colon carcinogenesis. Gut, 2010, 235754.

Fearon ER (2011). Molecular genetics of colorectal cancer. Annual Review of Pathology: Mechanisms of Disease, 6 , 479-507.

Gianotti L, Morelli L, Galbiati F, et al (2010). A randomized double-blind trial on perioperative administration of probiotics in colorectal cancer patients. World journal of gastroenterology: WJG, 16, 167.

Guarner F, Malagelada J-R (2003). Gut flora in health and disease. The Lancet, 361, 512-9.

Hardwick JC, Van Den Brink GR, Offerhaus G, et al (2001). Leptin is a growth factor for colonic epithelial cells. Gastroenterology, 121, 79-90.

Hoda M, Keely S, Bertelsen L, et al (2007). Leptin acts as a mitogenic and antiapoptotic factor for colonic cancer cells. Br J Surg, 94, 346-54.

Holick MF (2010). Vitamin D and health: evolution, biologic functions, and recommended dietary intakes for vitamin D. In 'Vitamin D', Eds Springer, 3-33

Huang P, Chandra V, Rastinejad F (2010). Structural overview of the nuclear receptor superfamily: insights into physiology and therapeutics. Annu Rev Physiol, 72, 247-72.

Hui L, Desen W, Zhizhong P, et al (2011). Expression and biological significance of leptin, leptin receptor, VEGF, and CD34 in colorectal carcinoma. Cell Biochem Biophysics, 60, 241-4.

Ishikawa H, Akedo I, Otani T, et al (2005). Randomized trial of dietary fiber and Lactobacillus casei administration for prevention of colorectal tumors. Int J Cancer, 116, 762-7.

Ishikawa M, Kitayama J, Nagawa H (2004). Enhanced expression of leptin and leptin receptor (OB-R) in human breast cancer. Clin Cancer Res, 10, 4325-31.

Isono M, Inoue R, Kamida T, et al (2003). Significance of leptin expression in invasive potential of pituitary adenomas. Clin Neurol Neurosurgery, 105, 111-6.
Joshi RK, Lee S-A (2014). Obesity related adipokines and colorectal cancer: a review and meta-analysis. Asian Pac J Cancer Prev, 15, 397-405.

Kailasapathy K, Chin J (2000). Survival and therapeutic potential of probiotic organisms with reference to Lactobacillus acidophilus and Bifidobacterium spp. Immunology and Cell Biology, 78, 80-8.

Kaji R, Kiyoshima-Shibata J, Nagaoka M, et al (2010). Bacterial teichoic acids reverse predominant IL-12 production induced by certain lactobacillus strains into predominant IL-10 production via TLR2-dependent ERK activation in macrophages. J Immunol, 184, 3505-13.

Kotnis A, Sarin R, Mulherkar R (2005). Genotype, phenotype and cancer: role of low penetrance genes and environment in tumour susceptibility. J Biosciences, 30, 93-102.

Kumar M, Nagpal R, Verma V, et al (2013). Probiotic metabolites as epigenetic targets in the prevention of colon cancer. Nutr Rev, 71, 23-34.

Kumor A, Daniel P, Pietruczuk M, et al (2009). Serum leptin, adiponectin, and resistin concentration in colorectal adenoma and carcinoma (CC) patients. Int J Colorectal Disease, 24, 275-81.

Lagishetty V, Misharin AV, Liu NQ, et al (2010). Vitamin D deficiency in mice impairs colonic antibacterial activity and predisposes to colitis. Endocrinology, 151, 2423-32.

Lee NK, Park JS, Park E, et al (2007). Adherence and anticarcinogenic effects of Bacillus polyfermenticus SCD in the large intestine. Letters in Applied Microbiol, 44, 274-8.

Leung K-C (2004). Regulation of cytokine receptor signaling by nuclear hormone receptors: a new paradigm for receptor interaction. DNA and Cell Biology, 23, 463-74.

Lu R, Wu S, Xia Y, et al (2012). The vitamin D receptor, inflammatory bowel diseases, and colon cancer. Current Colorectal Cancer Reports, 8, 57-65.

Matilainen JM, Rasanen A, Gynther P, et al (2010). The genes encoding cytokines $<\mathrm{i}>\mathrm{IL}-2</ \mathrm{i}>,<\mathrm{i}>\mathrm{IL}-10</ \mathrm{i}>$ and $<\mathrm{i}>\mathrm{IL}-$ $12 \mathrm{~B}</ \mathrm{i}>$ are primary $1 \alpha, 25(\mathrm{OH})<\mathrm{sub}>2</$ sub $>\mathrm{D}<\mathrm{sub}>$ $3</$ sub $>$ target genes. J Steroid Biochemistry and Molecular Biology, 121, 142-5.

Matsunuma A, Horiuchi N (2007). Leptin attenuates gene expression for renal 25-hydroxyvitamin $\mathrm{D}<\mathrm{sub}>3<$ sub $>$ $1 \alpha$-hydroxylase in mice via the long form of the leptin receptor. Arch Biochem Biophys, 463, 118-27.

McEwan IJ 2009. Nuclear receptors: one big family, Springer.

Mencarelli A, Distrutti E, Renga B, et al (2011). Probiotics modulate intestinal expression of nuclear receptor and provide counter-regulatory signals to inflammation-driven adipose tissue activation. PLoS One, 6, 22978.

Menendez C, Lage M, Peino R, et al (2001). Retinoic acid and vitamin $\mathrm{D}$ (3) powerfully inhibit in vitro leptin secretion by human adipose tissue. J Endocrinol, 170, 425-31.

Mirza NN, McCloud JM, Cheetham MJ (2009). Clostridium septicum sepsis and colorectal cancer-a reminder. World $J$ Surg Oncol, 7, 73.

Mu H-J, Zou J, Xie P, et al (2014). Association of leptin receptor Lys109Arg and Gln223Arg polymorphisms with increased risk of clear cell renal cell carcinoma. Asian Pac J Cancer Prev, 15, 4211-5.

O'mahony L, Feeney M, O'halloran S, et al (2001). Probiotic impact on microbial flora, inflammation and tumour development in IL-10 knockout mice. Alimentary Pharmacology \& Therapeutics, 15, 1219-25.

Palmer HG, Gonzalez-Sancho JM, Espada J, et al (2001). Vitamin $\mathrm{D}(3)$ promotes the differentiation of colon carcinoma cells by the induction of E-cadherin and the inhibition of betacatenin signaling. J Cell Biol, 154, 369-87.

Pendas-Franco N, Aguilera O, Pereira F, et al (2008). Vitamin 
$\mathrm{D}$ and $\mathrm{Wnt} / \beta$-catenin pathway in colon cancer: role and regulation of DICKKOPF genes. Anticancer Research, 28, 2613-23.

Pereira F, Larriba MJ, Muñoz A (2012). Vitamin D and colon cancer. Endocrine-related cancer, 19, 51-71.

Ramagopalan SV, Heger A, Berlanga AJ, et al (2010). AChIP-seq defined genome-wide map of vitamin $\mathrm{D}$ receptor binding: associations with disease and evolution. Genome Research, 20, 1352-60.

Rasool S, Kadla SA, Khan T, et al (2013). Association of a VDR gene polymorphism with risk of colorectal cancer in Kashmir. Asian Pac J Cancer Prev, 14, 5833-7.

Reiff C, Delday M, Rucklidge G, et al (2009). Balancing inflammatory, lipid, and xenobiotic signaling pathways by VSL\# 3, a biotherapeutic agent, in the treatment of inflammatory bowel disease. Inflammatory Bowel Diseases, 15, 1721-36.

Reya T, Clevers H (2005). Wnt signalling in stem cells and cancer. Nature, 434, 843-50.

Sălăgeanu A (2010). Serum levels of adipokines resistin and leptin in patients with colon cancer. J Med Life, $\mathbf{3}, 416$.

Samuel S, Sitrin MD (2008). Vitamin D's role in cell proliferation and differentiation. Nutrition reviews, 66, 116-24.

Schauber J, Dorschner RA, Coda AB, et al (2007). Injury enhances TLR2 function and antimicrobial peptide expression through a vitamin $\mathrm{D}$-dependent mechanism. The J Clin Invest, 117, 803-11.

Sentongo TA, Semaeo EJ, Stettler N, et al (2002). Vitamin D status in children, adolescents, and young adults with Crohn disease. Am J Clin Nutr, 76, 1077-81.

Shamsuzzaman AS, Winnicki M, Wolk R, et al (2004). Independent association between plasma leptin and C-reactive protein in healthy humans. Circulation, 109, 2181-5.

Slattery M, Ballard-Barbash R, Edwards S, et al (2003). Body mass index and colon cancer: an evaluation of the modifying effects of estrogen (United States). Cancer Causes Control, 14, 75-84.

Slattery ML, Curtin K, Sweeney C, et al (2007). Diet and lifestyle factor associations with $\mathrm{CpG}$ island methylator phenotype and BRAF mutations in colon cancer. Int $J$ Cancer, 120, 656-63.

Slattery ML, Wolff RK, Herrick J, et al (2008). Leptin and leptin receptor genotypes and colon cancer: Gene-gene and genelifestyle interactions. Int J Cancer, 122, 1611-7.

Sonoda J, Pei L, Evans RM (2008). Nuclear receptors: decoding metabolic disease. FEBS letters, 582, 2-9.

Stubbins RE, Hakeem A, Nunez NP (2012). Using components of the vitamin $\mathrm{D}$ pathway to prevent and treat colon cancer. Nutr Rev, 70, 721-9.

Sun J (2010). Vitamin D and mucosal immune function. Curr Opinion Gastroenterology, 26, 591.

Suzuki T, Franchi L, Toma C, et al (2007). Differential regulation of caspase- 1 activation, pyroptosis, and autophagy via Ipaf and ASC in Shigella-infected macrophages. PLoS pathogens, 3, 111 .

Thorne J, Campbell MJ (2008). The vitamin D receptor in cancer. Proc Nutrition Society, 67, 115-27.

Tutino V, Notarnicola M, Guerra V, et al (2011). Increased soluble leptin receptor levels are associated with advanced tumor stage in colorectal cancer patients. Anticancer Res, 31, 3381-3.

Uchiyama T, Takahashi H, Endo H, et al (2011). Role of the long form leptin receptor and of the STAT3 signaling pathway in colorectal cancer progression. Int J Oncol, 39, 935.

Urbanska AM, Bhathena J, Martoni C, et al (2009). Estimation of the potential antitumor activity of microencapsulated
Lactobacillus acidophilus yogurt formulation in the attenuation of tumorigenesis in Apc (Min/+) mice. Dig Dis Sci, 54, 264-73.

Wada K, Tanaka H, Maeda K, et al (2009). Vitamin D receptor expression is associated with colon cancer in ulcerative colitis. Oncology reports, 22, 1021.

Weiss ST (2011). Bacterial components plus vitamin D: the ultimate solution to the asthma (autoimmune disease) epidemic? J Allergy Clin Immunol Pract, 127, 1128.

Wu S, Liao AP, Xia Y, et al (2010). Vitamin D receptor negatively regulates bacterial-stimulated NF- $\chi \mathrm{B}$ activity in intestine. Am J Pathol, 177, 686-97.

Wu S, Sun J (2011). Vitamin D, vitamin D receptor, and macroautophagy in inflammation and infection. Discovery Med, 11, 325.

Yoon S, Wu S, Zhang Y-g, et al (2011). Probiotic regulation of vitamin D receptor in intestinal inflammation. Gastroenterology, 140, S-19.

Yoon SS, Sun J (2011). Probiotics, nuclear receptor signaling, and anti-inflammatory pathways. Gastroenterology research and practice, 2011.

Yu K, Yang J, Jiang Y, et al (2014). Vitamin D receptor BsmI polymorphism and colorectal cancer risk: an updated analysis. Asian Pac J Cancer Prev, 15, 4801-7.

Zheng W, Wong KE, Zhang Z, et al (2012). Inactivation of the vitamin D receptor in $\mathrm{APCmin} /+$ mice reveals a critical role for the vitamin D receptor in intestinal tumor growth. Int $J$ Cancer, 130, 10-9.

Zhu Y, Michelle Luo T, Jobin C, et al (2011). Gut microbiota and probiotics in colon tumorigenesis. Cancer Lett, 309, 119-27.

Zinser GM, Suckow M, Welsh J (2005). Vitamin D receptor (VDR) ablation alters carcinogen-induced tumorigenesis in mammary gland, epidermis and lymphoid tissues. J Steroid Biochem Mol Biol, 97, 153-64. 